\title{
Monogenic Pisot and Anti-Pisot Polynomials
}

\author{
Lenny Jones
}

\begin{abstract}
A Pisot number is a real algebraic integer $\alpha>1$ such that all of its Galois conjugates, other than $\alpha$ itself, lie inside the unit circle. An anti-Pisot number is a real algebraic integer $\alpha>1$, such that exactly one Galois conjugate of $\alpha$ lies inside the unit circle, and $\alpha$ has at least one Galois conjugate, other than $\alpha$ itself, outside the unit circle. We call the minimal polynomials of these algebraic integers, respectively, Pisot and anti-Pisot polynomials. In this article, we find infinite families of Pisot (anti-Pisot) polynomials $f(x)$ such that $\left\{1, \alpha, \alpha^{2}, \ldots, \alpha^{n-1}\right\}$ is a basis for the ring of integers of $\mathbb{Q}(\alpha)$, where $\alpha$ is a Pisot (anti-Pisot) number and $\operatorname{deg}(f)=n$, for certain $n$. We refer to these polynomials as monogenic Pisot (anti-Pisot) polynomials.
\end{abstract}

\section{Introduction}

A Pisot (or Pisot-Vijayaraghavan) number [1] is a real algebraic integer $\alpha>1$ such that all other zeros of the minimal polynomial $f(x) \in \mathbb{Z}[x]$ of $\alpha$ lie inside the unit circle. We call $f(x)$ a Pisot polynomial. Examples of Pisot numbers include the integers larger than 1 and the Golden Ratio $\phi=(1+\sqrt{5}) / 2$. Although Pisot numbers were known prior to 1920 [1], it was not until the publication of Pisot's thesis [21] in 1938 that widespread interest ensued. In 1944, Siegel [26] proved that the smallest Pisot number (also known as the plastic number 20]) is the real zero of $x^{3}-x-1$. Shortly before Siegel's paper, Salem 22 ] proved the remarkable fact that the set of all Pisot numbers is closed. Aside from their many interesting intrinsic properties, Pisot numbers have found applications in harmonic analysis [13], Fourier analysis [23, dynamical systems [24] and quasicrystals [14, 15.

An anti-Pisot number 25] is a real algebraic integer $\alpha>1$, such that exactly one of the Galois conjugates of $\alpha$ lies inside the unit circle, and $\alpha$ has at least one Galois conjugate, other than $\alpha$ itself, outside the unit circle. We refer to the minimal polynomial of an antiPisot number as an anti-Pisot polynomial. Anti-Pisot numbers were used by Sidorov and Solomyak 25 to study when certain sets related to anti-Pisot polynomials with coefficients in $\{-1,0,1\}$ are dense in $\mathbb{R}$. Noting that if $\alpha \in(1,2)$, with $\alpha \neq(1+\sqrt{5}) / 2$, has a real conjugate $\beta$ such that $1 /|\beta|$ is a Pisot number, then $\alpha$ is an anti-Pisot number, Hare and

Received March 18, 2021; Accepted October 21, 2021.

Communicated by Ming-Lun Hsieh.

2020 Mathematics Subject Classification. Primary: 11R06; Secondary: 12F05, 11R09.

Key words and phrases. Pisot, anti-Pisot, monogenic, irreducible polynomial. 
Sidorov [8] have recently utilized anti-Pisot numbers in their study of conjugates of Pisot numbers.

Throughout this article, unless stated otherwise, when we say $f(x) \in \mathbb{Z}[x]$ is "irreducible" or "reducible", we mean over the rational numbers $\mathbb{Q}$. Let $f(x) \in \mathbb{Z}[x]$ be monic and irreducible with $\operatorname{deg}(f)=n$. Suppose that $f(\theta)=0$. Let $K=\mathbb{Q}(\theta)$ and let $\mathbb{Z}_{K}$ denote the ring of integers of $K$. We then have the well-known equation [4]

$$
\Delta(f)=\left[\mathbb{Z}_{K}: \mathbb{Z}[\theta]\right]^{2} \Delta(K)
$$

where $\Delta(*)$ denotes the discriminant of $*$. We say a monic polynomial $f(x) \in \mathbb{Z}[x]$ is monogenic if $f(x)$ is irreducible and $\left[\mathbb{Z}_{K}: \mathbb{Z}[\theta]\right]=1$; or, equivalently from (1.1), that $\Delta(f)=\Delta(K)$. Thus, $f(x)$ is monogenic if and only if

$$
\left\{1, \theta, \theta^{2}, \ldots, \theta^{n-1}\right\} \text { is a basis for } \mathbb{Z}_{K}
$$

The basis in $(1.2)$ is called a power basis, which facilitates calculations in $\mathbb{Z}_{K}$. Observe from (1.1) that if $\Delta(f)$ is squarefree, then $f(x)$ is monogenic.

The work in this article was motivated by [3,28,29], and, in particular, by the following theorem of Cheng and Zhuang [3]:

Theorem 1.1. Let $K$ be a real Galois extension over $\mathbb{Q}$ given by its integral basis $\beta_{1}, \beta_{2}$, $\ldots, \beta_{n}$. There exists a polynomial time algorithm to determine integers $a_{1}, a_{2}, \ldots, a_{n}$ such that

$$
\alpha=a_{1} \beta_{1}+a_{2} \beta_{2}+\cdots+a_{n} \beta_{n},
$$

where $\alpha$ is a Pisot number and $K=\mathbb{Q}(\alpha)$.

Thus, given a Pisot (or anti-Pisot) number $\alpha$ whose Pisot (or anti-Pisot) polynomial $f(x)$ has degree $n$, it is somewhat natural to ask if $f(x)$ is monogenic. That is,

Question 1.2. When is $\mathcal{B}=\left\{1, \alpha, \alpha^{2}, \ldots, \alpha^{n-1}\right\}$ a basis for $\mathbb{Z}_{K}$ ?

Since $\alpha^{k}$ is a Pisot number for any integer $k \geq 1[1]$, we make the interesting observation that if $\mathcal{B}$ is in fact a basis for $\mathbb{Z}_{K}$, then $\mathbb{Z}_{K}$ possesses a basis in which every element, other than 1 , is a Pisot number. We say that the minimal polynomial $f(x)$ for the Pisot number $\alpha$ is a monogenic Pisot polynomial when $\mathbb{Z}_{K}$ has the power basis $\mathcal{B}$. We define a monogenic anti-Pisot polynomial similarly.

In this article, we provide a partial answer to Question 1.2 by finding sufficient conditions for the monogenity of certain classes of Pisot polynomials, as well as proving the existence of infinite families of monogenic Pisot and anti-Pisot polynomials. More precisely, we prove the following: 
Theorem 1.3. Let $n$ and $t$ be integers with $n \geq 2$ and $t \geq 1$. Let

$$
f_{n, t}(x)=x^{n}-t\left(\frac{x^{n}-1}{x-1}\right) .
$$

(1) Suppose that $t \geq 2$. If

$$
t \quad \text { and } \quad \frac{(n+1)^{n+1} t-n^{n}(t+1)^{n+1}}{(1-n t)^{2}}
$$

are squarefree, then $f_{n, t}(x)$ is a monogenic Pisot polynomial.

(2) Suppose that $t=1$ and $n=2^{m}-1$ for some integer $m \geq 2$. If

$$
\frac{\left(2^{m}-1\right)^{2^{m}-1}-2^{2^{m}(m-1)}}{\left(2^{m-1}-1\right)^{2}}
$$

is squarefree, then $f_{n, 1}(x)$ is a monogenic Pisot polynomial.

Theorem 1.4. Let $n$ and $t$ be integers with $n \geq 5$ and $1 \leq|t| \leq n-4$. Suppose that either $|t|=1$ or $\left(\frac{(n-3)(n+1)}{p}\right)=-1$ for each prime divisor $p$ of $t$, where $\left(\frac{*}{p}\right)$ denotes the Legendre symbol. Then

$$
f(x)=x^{n}-(n-1) x^{n-1}+x^{n-2}+t
$$

is a Pisot polynomial, and $f(x)$ is monogenic if

$$
t \quad \text { and } \quad\left(n^{n} t+n+1\right)\left(t-(n-3)(n-2)^{n-2}\right)
$$

are squarefree.

The next theorem proves the existence, in various situations, of infinite families of monogenic Pisot and anti-Pisot polynomials.

Theorem 1.5. In each of the following situations, there exist infinitely many prime values of the indeterminate $t$ such that

(1) $x^{3}-t\left(x^{2}+a+1\right)$ is a monogenic Pisot polynomial when $a \in\{0,2\}$,

(2) $x^{n}-t\left(\frac{x^{n}-1}{x-1}\right)$ is a monogenic Pisot polynomial when $n \in\{2,4\}$,

(3) $x^{n}-16 c^{n-1} n(n t+w) x^{n-1}+n t+w$ is a monogenic Pisot polynomial when $c$ is a positive integer and $(n, w) \in\{(3,1),(4,2),(5,1),(7,1),(9,2)\}$,

(4) $x^{n}-t x-t$ is a monogenic anti-Pisot polynomial when $n \equiv 0(\bmod 2)$,

(5) $x^{n}-t\left(x^{2}+(n-1) x+1\right)$ is a monogenic anti-Pisot polynomial when $n \geq 4$, 
(6) $x^{n}-t\left(x^{3}+\left(\frac{5 n+1}{2}\right) x^{2}+\left(\frac{3 n^{2}+3 n-4}{2}\right) x+\frac{3 n^{2}-5 n+4}{2}\right)$ is a monogenic anti-Pisot polynomial when $n \geq 5$ with $n \equiv 1(\bmod 4)$,

(7) $x^{n}-t\left(x^{3}+(2 n+1) x^{2}+\left(n^{2}+2 n-1\right) x+n^{2}-n+1\right)$ is a monogenic anti-Pisot polynomial when $n \geq 4$ with $n \equiv 0(\bmod 2)$ and $n \not \equiv 10(\bmod 14)$.

It turns out that all polynomials $f(x)$ in Theorem 1.5 have the property that the largest degree of any irreducible factor of $\Delta(f)$ in the indeterminate $t$ is at most 3 . This fact allows us to achieve the results of Theorem 1.5 unconditionally. In Theorem 1.5(1), (2) and (3), more general results are attainable if we allow the degree of an irreducible factor of $\Delta(f)$ to be larger than 3. However, in this situation, the conclusions are conditional on the $a b c$-conjecture for number fields (see Theorem 2.7 and Corollary 2.8).

Remarks 1.6. (1) When $t=1$, the polynomials $f_{n, 1}(x)$ in 1.3 are characteristic polynomials of generalized Fibonacci recurrence sequences, and the Pisot numbers that are zeros of $f_{n, 1}(x)$ are known as Littlewood Pisot numbers 16.

(2) The Pisot numbers $\alpha$ in Theorems 1.3 and 1.4 are units in $\mathbb{Z}_{\mathbb{Q}(\alpha)}$ whenever $|t|=1$.

(3) The existence of infinitely many prime values of $t$, such that the polynomials in Theorem 1.5(5) and (6) of are monogenic, was established in 12.

\section{Preliminaries}

The formula for the discriminant of an arbitrary monic trinomial, due to Swan [27], is given in the following theorem.

Theorem 2.1. Let $f(x)=x^{n}+A x^{m}+B \in \mathbb{Q}[x]$, where $0<m<n$, and let $d=\operatorname{gcd}(n, m)$. Then

$$
\Delta(f)=(-1)^{n(n-1) / 2} B^{m-1}\left(n^{n / d} B^{(n-m) / d}-(-1)^{n / d}(n-m)^{(n-m) / d} m^{m / d} A^{n / d}\right)^{d} .
$$

The next theorem is due to Perron 19 .

Theorem 2.2. Let $n \geq 2$ and let

$$
f(x)=x^{n}+a_{n-1} x^{n-1}+a_{n-2} x^{n-2}+\cdots+a_{1} x+a_{0} \in \mathbb{C}[x]
$$

with $a_{0} \neq 0$, be such that

$$
\left|a_{n-1}\right|>1+\left|a_{n-2}\right|+\left|a_{n-3}\right|+\cdots+\left|a_{0}\right|
$$

Then $f(x)$ has exactly $n-1$ zeros inside the unit circle. Furthermore, if $f(x) \in \mathbb{Z}[x]$, then $f(x)$ is irreducible. 
We then have the following corollary.

Corollary 2.3. Let $f(x)$ be a polynomial satisfying the hypotheses of Theorem 2.2. If $f(1)<0$, then $f(x)$ is a Pisot polynomial.

Proof. By Theorem 2.2, $f(x)$ is irreducible and has exactly one zero $\alpha$ with $|\alpha| \geq 1$. Hence, we deduce that $\alpha \in \mathbb{R}$ and $|\alpha|>1$. Since the leading coefficient of $f(x)$ is positive, and $f(1)<0$, it follows that $\alpha>1$. Thus, $f(x)$ is a Pisot polynomial.

The next theorem is a standard tool used to determine if an irreducible polynomial is monogenic.

Theorem 2.4. (Dedekind's Criterion [4]) Let $\theta$ be an algebraic integer, and let $K$ denote the number field $\mathbb{Q}(\theta)$ with ring of integers $\mathbb{Z}_{K}$. Let $T(x) \in \mathbb{Z}[x]$ be the monic minimal polynomial of $\theta$. Let $p$ be a prime number and let $₹$ denote reduction of $*$ modulo $p$ (in $\mathbb{Z}$, $\mathbb{Z}[x]$ or $\mathbb{Z}[\theta])$. Let

$$
\bar{T}(x)=\prod_{i=1}^{s} \overline{\gamma_{i}}(x)^{e_{i}}
$$

be the factorization of $T(x)$ modulo $p$ in $\mathbb{F}_{p}[x]$, and set

$$
g(x)=\prod_{i=1}^{s} \gamma_{i}(x),
$$

where the $\gamma_{i}(x) \in \mathbb{Z}[x]$ are arbitrary monic lifts of the $\overline{\gamma_{i}}(x)$. Let $h(x) \in \mathbb{Z}[x]$ be a monic lift of $\bar{T}(x) / \bar{g}(x)$ and set

$$
F(x)=\frac{g(x) h(x)-T(x)}{p} \in \mathbb{Z}[x]
$$

Then

$$
\left[\mathbb{Z}_{K}: \mathbb{Z}[\theta]\right] \not \equiv 0 \quad(\bmod p) \Longleftrightarrow \operatorname{gcd}(\bar{F}, \bar{g}, \bar{h})=1 \quad \text { in } \mathbb{F}_{p}[x]
$$

Theorem 2.5. [5] Let $p$ be a prime and let $f(x) \in \mathbb{Z}[x]$ be a monic $p$-Eisenstien polynomial with $\operatorname{deg}(f)=n$. Let $K=\mathbb{Q}(\theta)$, where $f(\theta)=0$. Then

(1) $p^{n-1} \| \Delta(K)$ if $n \not \equiv 0(\bmod p)$,

(2) $p^{n} \mid \Delta(K)$ if $n \equiv 0(\bmod p)$.

The following theorem of Jakhar, Khanduja and Sangwan gives necessary and sufficient conditions for a monic irreducible trinomial to be monogenic. It can be thought of as a more "streamlined" version of Theorem 2.4 in this special case. 
Theorem 2.6. [11] Let $n \geq 2$ be an integer. Let $K=\mathbb{Q}(\theta)$ be an algebraic number field with $\theta \in \mathbb{Z}_{K}$, the ring of integers of $K$, having minimal polynomial $f(x)=x^{n}+A x^{m}+B$ over $\mathbb{Q}$, where $\operatorname{gcd}(m, n)=d_{0}, m=m_{1} d_{0}$ and $n=n_{1} d_{0}$. A prime factor $q$ of $\Delta(f)$ does not divide $\left[\mathbb{Z}_{K}: \mathbb{Z}[\theta]\right]$ if and only if $q$ satisfies one of the following conditions:

(i) when $q \mid A$ and $q \mid B$, then $q^{2} \nmid B$;

(ii) when $q \mid A$ and $q \nmid B$, then

$$
\text { either } q \mid a_{2} \text { and } q \nmid b_{1} \text { or } q \nmid a_{2}\left((-B)^{m_{1}} a_{2}^{n_{1}}+\left(-b_{1}\right)^{n_{1}}\right) \text {, }
$$

where $a_{2}=A / q$ and $b_{1}=\frac{B+(-B)^{q^{j}}}{q}$, such that $q^{j} \| n$ with $j \geq 1$;

(iii) when $q \nmid A$ and $q \mid B$, then

$$
\text { either } q \mid a_{1} \text { and } q \nmid b_{2} \text { or } q \nmid a_{1} b_{2}^{m-1}\left((-A)^{m_{1}} a_{1}^{n_{1}-m_{1}}-\left(-b_{2}\right)^{n_{1}-m_{1}}\right) \text {, }
$$

where $a_{1}=\frac{A+(-A)^{q^{l}}}{q}$, such that $q^{l} \|(n-m)$ with $l \geq 0$, and $b_{2}=B / q$;

(iv) when $q \nmid A B$ and $q \mid m$ with $n=s^{\prime} q^{k}, m=s q^{k}, q \nmid \operatorname{gcd}\left(s^{\prime}, s\right)$, then the polynomials

$$
x^{s^{\prime}}+A x^{s}+B \text { and } \frac{A x^{s q^{k}}+B+\left(-A x^{s}-B\right)^{q^{k}}}{q}
$$

are coprime modulo q;

(v) when $q \nmid A B m$, then

$$
q^{2} \nmid\left(B^{n_{1}-m_{1}} n_{1}^{n_{1}}-(-1)^{m_{1}} A^{n_{1}} m_{1}^{m_{1}}\left(m_{1}-n_{1}\right)^{n_{1}-m_{1}}\right) .
$$

Combining work of Helfgott 9 and Pasten [18], with earlier work of [10], we arrive at the following "state of affairs" with regard to squarefree values of polynomials at prime arguments.

Theorem 2.7. Let $f(x) \in \mathbb{Z}[x]$, and suppose that $f(x)$ factors into a product of distinct irreducibles, where the largest degree of any irreducible factor of $f(x)$ is d. Define

$$
N_{f}(X)=\mid\{p \leq X: p \text { is prime and } f(p) \text { is squarefree }\} \mid .
$$

Then, the following asymptotic holds unconditionally if $d \leq 3$, and holds, assuming the abc-conjecture for number fields for $f(x)$, if $d \geq 4$ :

$$
N_{f}(X) \sim c_{f} \frac{X}{\log (X)},
$$

where

$$
c_{f}=\prod_{r \text { prime }}\left(1-\frac{\rho_{f}\left(r^{2}\right)}{r(r-1)}\right)
$$

and $\rho_{f}\left(r^{2}\right)$ is the number of $z \in\left(\mathbb{Z} / r^{2} \mathbb{Z}\right)^{*}$ such that $f(z) \equiv 0\left(\bmod r^{2}\right)$. 
The unconditional part of the following immediate corollary of Theorem 2.7 is a main tool used in the proof of Theorem 1.5 .

Corollary 2.8. Let $f(x) \in \mathbb{Z}[x]$, and suppose that $f(x)$ factors into a product of distinct irreducibles, where the largest degree of any irreducible factor of $f(x)$ is $d$. We suppose further that, for each prime $r$, there exists some $z \in\left(\mathbb{Z} / r^{2} \mathbb{Z}\right)^{*}$ such that $f(z) \not \equiv 0\left(\bmod r^{2}\right)$. If $d \leq 3$, or if $d \geq 4$ and assuming the abc-conjecture for number fields for $f(x)$, then there exist infinitely many primes $p$ such that $f(p)$ is squarefree.

Remark 2.9. The assumption that, for each prime $r$, there exists some $z \in\left(\mathbb{Z} / r^{2} \mathbb{Z}\right)^{*}$ such that $f(z) \not \equiv 0\left(\bmod r^{2}\right)$, is made in Corollary 2.8 to avoid the situation that $c_{f}=0$ in Theorem 2.7

Definition 2.10. If $f(z) \equiv 0\left(\bmod r^{2}\right)$, for some prime $r$ and all $z \in\left(\mathbb{Z} / r^{2} \mathbb{Z}\right)^{*}$, then we say that $f(x)$ has an obstruction at $r$.

The following proposition contains a new discriminant formula that is useful to us here.

Proposition 2.11. Let $n$ and $t$ be integers with $n \geq 2$. Let

$$
f(x)=x^{n}-t\left(\frac{x^{n}-1}{x-1}\right)=x^{n}-t\left(x^{n-1}+x^{n-2}+\cdots+x+1\right) .
$$

Then

$$
\Delta(f)=\frac{(-1)^{(n+1) n / 2} t^{n-1}\left((n+1)^{n+1} t-n^{n}(t+1)^{n+1}\right)}{(1-n t)^{2}} .
$$

Proof. Observe that

$$
(x-1) f(x)=x^{n+1}-(t+1) x^{n}+t .
$$

Using familiar properties of the discriminant, we see that

$$
\Delta((x-1) f(x))=\Delta(x-1) \Delta(f) R(x-1, f)^{2}=\Delta(f)(1-n t)^{2},
$$

where $R$ is the resultant. By Theorem 2.1, we have that

$$
\begin{aligned}
& \Delta\left(x^{n+1}-(t+1) x^{n}+t\right) \\
= & (-1)^{(n+1) n / 2} t^{n-1}\left((n+1)^{n+1} t-(-1)^{n+1} n^{n}(-(t+1))^{n+1}\right) .
\end{aligned}
$$

Equating 2.1) and 2.2, and solving for $\Delta(f)$ completes the proof.

We require the following additional discriminant formula, which appears in 12 . 
Theorem 2.12. Let $n$ and $k$ be integers with $n>k \geq 1$. Let

$$
f(x)=x^{n}+\operatorname{tg}(x)
$$

where $t \in \mathbb{Q}$ and

$$
g(x)=a_{k} x^{k}+a_{k-1} x^{k-1}+a_{k-2} x^{k-2}+\cdots+a_{1} x+a_{0} \in \mathbb{Z}[x]
$$

with $a_{0} a_{k} \neq 0$. Define

$$
\widehat{g}(x):=a_{k}(n-k) x^{k}+a_{k-1}(n-(k-1)) x^{k-1}+\cdots+a_{1}(n-1) x+a_{0} n,
$$

and suppose that

$$
\widehat{g}(x)=\prod_{i=1}^{k}\left(A_{i} x+B_{i}\right)
$$

where the $A_{i} x+B_{i} \in \mathbb{Z}[x]$ are not necessarily distinct. If $f(x)$ is irreducible, then

$$
\Delta(f)=\frac{(-1)^{\frac{n(n+2 k-1)}{2}} t^{n-1} \prod_{i=1}^{k}\left(\left(-B_{i}\right)^{n}+t \sum_{j=0}^{k} a_{j} A_{i}^{n-j}\left(-B_{i}\right)^{j}\right)}{a_{0}} .
$$

Remark 2.13. Note that $f(x)$ is a trinomial in Theorem 2.12 when $k=1$, and Swan's formula in Theorem 2.1 with $m=1$ is recovered in this situation.

We require the next lemma for the proof of Theorem 1.3 .

Lemma 2.14. Let $m \geq 2$ be an integer. Then, for $j$ with $1 \leq j \leq 2^{m}-1$,

$$
\text { [7. }\left(\begin{array}{c}
2^{m} \\
j
\end{array}\right) \equiv \begin{cases}2(\bmod 4) & \text { if } j=2^{m-1} \\
0(\bmod 4) & \text { otherwise }\end{cases}
$$

(2) $\left(\begin{array}{c}2^{m}-1 \\ j\end{array}\right) \equiv 1(\bmod 2)$.

Remark 2.15. Lemma 2.14(2) follows directly from a theorem of Lucas on binomial coefficients modulo $p$. A statement and proof of this theorem can be found in [6].

\section{Proof of Theorem 1.3}

Proof of Theorem 1.3. For any $n \geq 2$ and $t \geq 1$, the fact that $f_{n, t}(x)$ in $(1.3)$ is a Pisot polynomial follows from [2, Theorem 2]. Next, we address the monogenity of $f_{n, t}(x)$. Let

$$
D:=\frac{(n+1)^{n+1} t-n^{n}(t+1)^{n+1}}{(1-n t)^{2}} .
$$

Suppose that $f_{n, t}(\alpha)=0$, and let $K=\mathbb{Q}(\alpha)$. 
We begin with Theorem 1.3(1). Let $p$ be a prime divisor of $t$. Since $t$ is squarefree, we have that $f_{n, t}(x)$ is $p$-Eisenstein, and is therefore irreducible. Moreover, $\operatorname{gcd}(t, D) \equiv 0$ $(\bmod p)$ if and only if $n \equiv 0(\bmod p)$, and in this case, we have that $p \| D$ since $D$ is squarefree. Therefore, it follows from Proposition 2.11 and Theorem 2.5 that $\Delta(K)=$ $\Delta\left(f_{n, t}\right)$, and hence, $f_{n, t}(x)$ is monogenic.

We turn now to Theorem 1.3(2). Since $n=2^{m}-1$ and $t=1$, we see that

$$
D=\frac{2^{2^{m}-2}\left(2^{2^{m}}(m-1)-\left(2^{m}-1\right)^{2^{m}-1}\right)}{\left(1-2^{m-1}\right)^{2}} \equiv 0 \quad(\bmod 4)
$$

since $m \geq 2$. Hence, $D$ is never squarefree in this situation, and Theorem $1.3(1)$ is ineffective in determining whether $f_{n, 1}(x)$ is monogenic. To establish this part of the theorem, we use Theorem 2.4 with $p=2$ to show that

$$
\left[\mathbb{Z}_{K}: \mathbb{Z}[\alpha]\right] \not \equiv 0 \quad(\bmod 2) .
$$

Since discriminants are translation invariant, we can use $T(x):=f_{n, 1}(x+1)$ in place of $f_{n, t}(x)$ in Theorem 2.4 to show $(3.2)$. To see how $T(x)$ factors modulo 2 into irreducibles, we define

$$
\mathcal{F}(x):=(x-1) f_{n, 1}(x)=x^{n+1}-2 x^{n}+1=x^{2^{m}}-2 x^{2^{m}-1}+1
$$

so that

$$
\begin{aligned}
T(x) & =\frac{\mathcal{F}(x+1)}{x}=\frac{(x+1)^{2^{m}}-2(x+1)^{2^{m}-1}+1}{x} \\
& =\sum_{j=1}^{2^{m}}\left(\begin{array}{c}
2^{m} \\
j
\end{array}\right) x^{j-1}-2 \sum_{j=1}^{2^{m}-1}\left(\begin{array}{c}
2^{m}-1 \\
j
\end{array}\right) x^{j-1} .
\end{aligned}
$$

Then, by Lemma 2.14, we have that $\bar{T}(x)=x^{2^{m}-1}$. Hence, we may let $g(x)=x$ and $h(x)=x^{2^{m}-2}$ to calculate $F(x)$ in Theorem 2.4 .

$$
\begin{aligned}
F(x) & =\frac{g(x) h(x)-T(x)}{2}=\frac{x^{2^{m}-1}-\left(\sum_{j=1}^{2^{m}}\left(\begin{array}{c}
2^{m} \\
j
\end{array}\right) x^{j-1}-2 \sum_{j=1}^{2^{m}-1}\left(\begin{array}{c}
2^{m}-1 \\
j
\end{array}\right) x^{j-1}\right)}{2} \\
& =-\sum_{j=1}^{2^{m}-1} \frac{\left(\begin{array}{c}
2^{m} \\
j
\end{array}\right)}{2} x^{j-1}+\sum_{j=1}^{2^{m}-1}\left(\begin{array}{c}
2^{m}-1 \\
j
\end{array}\right) x^{j-1} .
\end{aligned}
$$

Then, by Lemma 2.14 .

$$
\bar{F}(x)=x^{2^{m}-2}+x^{2^{m}-3}+\cdots+x^{2^{m-1}}+x^{2^{m-1}-2}+x^{2^{m-1}-3}+\cdots+x+1 .
$$

Thus, $\operatorname{gcd}(\bar{F}, \bar{g})=1$, which establishes $(3.2)$. Consequently, if

$$
\frac{2^{2^{m}(m-1)}-\left(2^{m}-1\right)^{2^{m}-1}}{\left(1-2^{m-1}\right)^{2}}
$$

is squarefree, then $f(x)$ is monogenic.

Remark 3.1. Computer computations verify that $D$, as defined in (3.1), is squarefree when $t=1$ and $3 \leq n \leq 49$. 


\section{Proof of Theorem 1.4}

Proof of Theorem 1.4. We have that

$$
f(x)=x^{n}-(n-1) x^{n-1}+x^{n-2}+t \in \mathbb{Z}[x]
$$

with $1 \leq|t| \leq n-4$, such that either $|t|=1$ or $\left(\frac{(n-3)(n+1)}{p}\right)=-1$ for each prime divisor of $t$.

Using Corollary 2.3, straightforward calculations show that $f(x)$ is, in fact, a Pisot polynomial for any integer $t$ with $1 \leq|t| \leq n-4$.

Next, to establish the fact that $f(x)$ is monogenic if

$$
t \text { and }\left(n^{n} t+n+1\right)\left(t-(n-3)(n-2)^{n-2}\right) \quad \text { are squarefree, }
$$

we need to calculate $\Delta(f)$. To accomplish this task, we define

$$
\mathcal{F}(x):=\frac{\widetilde{f}(x)}{t}=x^{n}+\frac{1}{t}\left(x^{2}-(n-1) x+1\right),
$$

where $\tilde{f}$ denotes the reciprocal of $f$. Note that $\mathcal{F}(x)$ is irreducible since $f(x)$ is irreducible. Let $g(x)=x^{2}-(n-1) x+1$ and

$$
\widehat{g}(x)=(n-2) x^{2}-(n-1)^{2} x+n=(x-n)((n-2) x-1) .
$$

Then, we apply Theorem 2.12 to $\mathcal{F}(x)$, with

$$
a_{2}=a_{0}=1, \quad a_{1}=-(n+1), \quad A_{1}=1, \quad B_{1}=-n, \quad A_{2}=n-2 \quad \text { and } \quad B_{2}=-1,
$$

to get

$$
\begin{aligned}
\Delta(f) & =\Delta(\widetilde{f})=\Delta(t \mathcal{F})=t^{2 n-2} \Delta(\mathcal{F}) \\
& =t^{2 n-2}(-1)^{n(n+3) / 2} \frac{1}{t^{n-1}}\left(n^{n}+(n+1) \frac{1}{t}\right)\left(1-(n-3)(n-2)^{n-2} \frac{1}{t}\right) \\
& =(-1)^{n(n+3) / 2} t^{n-3}\left(n^{n} t+n+1\right)\left(t-(n-3)(n-2)^{n-2}\right) .
\end{aligned}
$$

Let $f(\alpha)=0$, and let $K=\mathbb{Q}(\alpha)$. Assume that conditions 4.1) hold. Note that if $|t|=1$, then

$$
\Delta(K) \equiv 0 \quad\left(\bmod \left(n^{n} t+n+1\right)\left(t-(n-3)(n-2)^{n-2}\right)\right)
$$

since $\left(n^{n} t+n+1\right)\left(t-(n-3)(n-2)^{n-2}\right)$ is squarefree. Hence, $\Delta(f)=\Delta(K)$, and $f(x)$ is monogenic in this case. Now suppose that $|t|>1$, and let $p$ be a prime divisor of $t$. Since $\left(\frac{(n-3)(n+1)}{p}\right)=-1$, it follows that $x^{2}-(n-1) x+1$ is irreducible over $\mathbb{F}_{p}$. Hence,

$$
f(x) \equiv x^{n-2}\left(x^{2}-(n-1) x+1\right) \quad(\bmod p)
$$


is the factorization of $f(x)$ into irreducibles over $\mathbb{F}_{p}$. We claim that $\left[\mathbb{Z}_{K}: \mathbb{Z}[\alpha]\right] \not \equiv 0$ $(\bmod p)$. Calculating $F(x)$ in Theorem 2.4 with

$$
T(x):=f(x), \quad g(x):=x\left(x^{2}-(n-1) x+1\right) \quad \text { and } \quad h(x):=x^{n-3},
$$

we see that

$$
\begin{aligned}
F(x) & =\frac{g(x) h(x)-T(x)}{p} \\
& =\frac{x^{n-2}\left(x^{2}-(n-1) x+1\right)-\left(x^{n}-(n-1) x^{n-1}+x^{n-2}+t\right)}{p}=\frac{-t}{p} .
\end{aligned}
$$

Thus, $\bar{F}$ is a nonzero constant since $t$ is squarefree. Therefore, $\operatorname{gcd}(\bar{F}, \bar{h})=1$ in $\mathbb{F}_{p}[x]$. Hence, by Theorem 2.4 , the claim that $\left[\mathbb{Z}_{K}: \mathbb{Z}[\alpha]\right] \not \equiv 0(\bmod p)$ is established. Consequently, as in the case of $|t|=1$, we have that $f(x)$ is monogenic since

$$
\left(n^{n} t+n+1\right)\left(t-(n-3)(n-2)^{n-2}\right)
$$

is squarefree.

Remark 4.1. In the proof of Theorem 1.4, a recent result of Otake and Shaska [17] can also be used to calculate $\Delta(\mathcal{F})$.

\section{Proof of Theorem 1.5}

Proof of Theorem 1.5. We give details only for Theorem 1.5 (2), (3) and (7), since many of the techniques are similar. Unless otherwise indicated, we assume that the domain for the indeterminate $t$ is the set of positive integers.

We begin with Theorem $1.5(2)$ where $f(x)=x^{n}-t\left(\frac{x^{n}-1}{x-1}\right)$. For arbitrary $n \geq 2$, the fact that $f(x)$ is a Pisot polynomial follows from results of Brauer [2]. We give details only for the case $n=4$, where in this case, we see that

$$
f(x)=x^{4}-t\left(x^{3}+x^{2}+x+1\right)
$$

since they are similar when $n=2$. Then, by Proposition 2.11,

$$
\Delta(f)=\frac{t^{3}\left(5^{5} t-4^{4}(t+1)^{5}\right)}{(1-4 t)^{2}}=-t^{3} \delta(t),
$$

where

$$
\delta(t)=16 t^{3}+88 t^{2}+203 t+256
$$

which is easily seen to be irreducible by the Rational Zero Theorem. We want to apply Corollary 2.8 to $\delta(t)$, so we must first show that $\delta(t)$ has no obstructions at any prime 
$r$ (see Definition 2.10). If, for some prime $r, \delta(z) \equiv 0\left(\bmod r^{2}\right)$ for all $z \in\left(\mathbb{Z} / r^{2} \mathbb{Z}\right)^{*}$, then $\delta(z) \equiv 0(\bmod r)$ for all $z \in(\mathbb{Z} / r \mathbb{Z})^{*}$. Hence, $\left|(\mathbb{Z} / r \mathbb{Z})^{*}\right|=r-1 \leq 3$ since $\delta(t)$ has at most 3 distinct zeros modulo $r$. Thus, we only have to check the primes $r \in\{2,3\}$. Since $\delta(1) \equiv 3(\bmod 4)$ and $\delta(1) \equiv 5(\bmod 9), \delta(t)$ has no obstructions at these primes. Hence, by Corollary 2.8, there exist (unconditionally) infinitely many primes $p$ such that $\delta(p)$ is squarefree. Let $p>2$ be such a prime, and let $\alpha$ be the unique Pisot zero of $f(x)=x^{4}-p\left(x^{3}+x^{2}+x+1\right)$. Let $K=\mathbb{Q}(\alpha)$, and let $\mathbb{Z}_{K}$ be the ring of integers of $K$. Since $\delta(p)$ is squarefree, we see from (5.1) that $\Delta(K) \equiv 0(\bmod \delta(p))$. Since $f(x)$ is $p$-Eisenstein and $\operatorname{gcd}(4, p)=1$, we have from Theorem 2.5 that $p^{3} \| \Delta(K)$. Thus, $|\Delta(f)|=|\Delta(K)|$ from (5.1), which completes the proof that $f(x)$ is monogenic.

Next, we address Theorem $1.5(3)$ where $f(x)=x^{n}-16 c^{n-1} n(n t+w) x^{n-1}+n t+w$. Since

$$
16 c^{n-1} n(n t+w)>n t+w \quad \text { and } \quad f(1)=1-16 c^{n-1} n(n t+w)+n t+w<0
$$

for any positive integer values of $c, t, w$ and $n$, it follows from Corollary 2.3 that $f(x)$ is a Pisot polynomial, and hence irreducible.

We have by Theorem 2.1 that

$$
\Delta(f)=(-1)^{\left(n^{2}-n+2\right) / 2} n^{n}(n t+w)^{n-2} D(t),
$$

where

$$
D(t)=(n t+w)\left(16^{n} c^{n(n-1)}(n-1)^{n-1}(n t+w)^{n-1}-1\right) .
$$

For each value of $n \in\{3,4,5,7,9\}$, we give the factored form of $\Delta(f)$ in Table 5.1 to see that no irreducible factor of $D(t)$ has degree larger than 3 .

\begin{tabular}{c|c}
$n$ & $\Delta(f)$ \\
\hline 3 & $3^{3} T^{2}\left(2^{7} c^{3} T-1\right)\left(2^{7} c^{3} T+1\right)$ \\
4 & $-2^{8} T^{3}\left(2^{16} 3^{3} c^{12} T^{3}-1\right)$ \\
5 & $-5^{5} T^{4}\left(2^{7} c^{5} T-1\right)\left(2^{7} c^{5} T+1\right)\left(2^{14} c^{10} T^{2}+1\right)$ \\
7 & $7^{7} T^{6}\left(2^{17} 3^{3} c^{21} T^{3}-1\right)\left(2^{17} 3^{3} c^{21} T^{3}+1\right)$ \\
9 & $-3^{18} T^{8}\left(2^{15} c^{18} T^{2}-1\right)\left(2^{15} c^{18} T^{2}+1\right)\left(2^{15} c^{18} T^{2}+2^{8} c^{9} T+1\right)\left(2^{15} c^{18} T^{2}-2^{8} c^{9} T+1\right)$
\end{tabular}

Table 5.1: Factored form of $\Delta(f)$ with $T:=n t+w$.

Since the arguments are similar for each value of $n \in\{3,4,5,7,9\}$, we give details only for $n=9$. Recall that $w=2$ in this case. We wish to apply Corollary 2.8 to the 
polynomial

$$
D(t)=(9 t+2)\left(2^{60} c^{72}(9 t+2)^{8}-1\right) .
$$

To do so, we must first show that $D(t)$ has no obstructions at any prime $r$. From 5.2 , we see that if $D(z) \equiv 0\left(\bmod r^{2}\right)$ for all $z \in\left(\mathbb{Z} / r^{2} \mathbb{Z}\right)^{*}$, then $D(z) \equiv 0(\bmod r)$ for all $z \in(\mathbb{Z} / r \mathbb{Z})^{*}$. Hence, $\left|(\mathbb{Z} / r \mathbb{Z})^{*}\right|=r-1 \leq 9$ since $D(t)$ has at most 9 distinct zeros modulo $r$. Thus, we have reduced the problem to showing that $D(t)$ has no obstructions at $r \in\{2,3,5,7\}$. Observe that $D(1) \equiv 1(\bmod 2)$, so that $D(t)$ has no obstructions at $r=2$. In Table 5.2, we give, for each $r \in\{3,5,7\}$ and every congruence class of $c$ $\left(\bmod r^{2}\right)$, values of integers $z$ with $\operatorname{gcd}(z, r)=1$ and the corresponding values of $D(z)$ $\left(\bmod r^{2}\right)$ for which $D(z) \not \equiv 0\left(\bmod r^{2}\right)$.

\begin{tabular}{c|c}
$r$ & {$\left[\left\{c\left(\bmod r^{2}\right)\right\}, z, D(z)\left(\bmod r^{2}\right)\right]$} \\
\hline 3 & {$[\{0, \pm 3\}, 1,7],[\{ \pm 1, \pm 2, \pm 4\}, 1,6]$} \\
\hline 5 & {$[\{0, \pm 5, \pm 10\}, 1,14],[\{ \pm 1, \pm 7\}, 1,5],[\{ \pm 2, \pm 11\}, 2,5]$,} \\
& {$[\{ \pm 3, \pm 4\}, 1,20],[\{ \pm 6, \pm 8\}, 1,15],[\{ \pm 9, \pm 12\}, 1,10]$} \\
\hline & {$[\{0, \pm 7, \pm 14, \pm 21\}, 1,38],[\{ \pm 1, \pm 18, \pm 19\}, 1,4],[\{ \pm 2, \pm 11, \pm 13\}, 1,25]$,} \\
& {$[\{ \pm 3, \pm 5, \pm 8\}, 1,18],[\{ \pm 4, \pm 22, \pm 23\}, 1,46],[\{ \pm 6, \pm 10, \pm 16\}, 1,39]$,} \\
& {$[\{ \pm 9, \pm 15, \pm 24\}, 1,32],[\{ \pm 12, \pm 17, \pm 20\}, 1,11]$}
\end{tabular}

Table 5.2: No obstructions for $D(t)$ at $r \in\{3,5,7\}$.

Therefore, we conclude from Corollary 2.8 that there exist (unconditionally, from Table 5.1 infinitely many primes $p$ such that $D(p)$ is squarefree. Let $p>3$ be such a prime, and let $\alpha$ be the unique Pisot zero of $f(x)=x^{9}-144 c^{8}(9 p+2) x^{8}+9 p+2$. Let $K=\mathbb{Q}(\alpha)$, and let $\mathbb{Z}_{K}$ be the ring of integers of $K$. Since $D(p)$ is squarefree, we see from Table 5.1 . that $\Delta(K) \equiv 0(\bmod D(p) /(9 p+2))$. To complete the proof that $f(x)$ is monogenic, we use Theorem 2.6 to show that $\left[\mathbb{Z}_{K}: \mathbb{Z}[\alpha]\right] \not \equiv 0(\bmod 3 q)$ for any prime $q$ dividing $9 p+2$. Applying Theorem 2.6 to $f(x)$, we have that $A=-144 c^{8}(9 p+2)$ and $B=9 p+2$. Since $9 p+2$ is squarefree, we see immediately that any prime $q$ dividing $9 p+2$ satisfies condition (i) of Theorem 2.6. Hence, $\left[\mathbb{Z}_{K}: \mathbb{Z}[\alpha]\right] \not \equiv 0(\bmod q)$. So, we now consider the prime 3. In this case, we have that $3 \mid A$ and $3 \nmid B$. Hence, we use condition (ii) of Theorem 2.6 . and since

$$
a_{2}=A / 3=-48 c^{8}(9 p+2) \quad \text { and } \quad b_{1}=\frac{(9 p+2)+(-(9 p+2))^{9}}{3} \equiv 1 \quad(\bmod 3),
$$

we see that $3 \mid a_{2}$ and $3 \nmid b_{1}$. Thus, we deduce that $\left[\mathbb{Z}_{K}: \mathbb{Z}[\alpha]\right] \not \equiv 0(\bmod 3)$, and therefore, $f(x)$ is a monogenic Pisot polynomial. 
Finally, we establish Theorem 1.5(7), where

$$
f(x)=x^{n}-t\left(x^{3}+(2 n+1) x^{2}+\left(n^{2}+2 n-1\right) x+n^{2}-n+1\right),
$$

such that $n \geq 4, n \equiv 0(\bmod 2)$ and $n \not \equiv 10(\bmod 14)$. Note that $f(x)$ is $p$-Eisenstein, and hence irreducible, if $t$ is a prime $p>n^{2}-n+1$.

We show now that $f(x)$ is an anti-Pisot polynomial when $t$ is a sufficiently large prime. The special case $n=4$ is handled first. In this situation, we have that

$$
f(x)=x^{4}-t x^{3}-9 t x^{2}-23 t x-13 t .
$$

When $t$ is a prime with $t>256$, we see from Table 5.3 that $f(x)$ has four real zeros $\rho_{1}$, $\rho_{2}, \rho_{3}$ and $\alpha$, where

$$
-5<\rho_{1}<-4, \quad-4<\rho_{2}<-3, \quad-1<\rho_{3}<0 \quad \text { and } \quad \alpha>1 .
$$

Thus, $\alpha$ is an anti-Pisot number and $f(x)$ is an anti-Pisot polynomial.

\begin{tabular}{c|ccccccc}
$z$ & -5 & -4 & -3 & -2 & -1 & 0 & 1 \\
\hline$f(z)$ & $2 t+625$ & $-t+256$ & $2 t+81$ & $5 t+16$ & $2 t+1$ & $-13 t$ & $-46 t+1$
\end{tabular}

Table 5.3: Values of $f(x)$ in (5.4).

Suppose now that $n \geq 6$, and define

$$
\begin{aligned}
F(x):= & \frac{-\widetilde{f}(x)}{t\left(n^{2}-n+1\right)} \\
= & x^{n}+\left(\frac{n^{2}+2 n-1}{n^{2}-n+1}\right) x^{n-1}+\left(\frac{2 n+1}{n^{2}-n+1}\right) x^{n-2} \\
& +\left(\frac{1}{n^{2}-n+1}\right) x+\frac{1}{t\left(n^{2}-n+1\right)}
\end{aligned}
$$

where

$$
\widetilde{f}(x)=-t\left(n^{2}-n+1\right) x^{n}-t\left(n^{2}+2 n-1\right) x^{n-1}-t(2 n+1) x^{n-2}-t x^{n-3}+1
$$

is the reciprocal of $f(x)$ in 5.3 . Note that if $t>0$, then the constant term and every coefficient of $F(x)$ are positive. Then

$$
\begin{aligned}
S & :=\frac{n^{2}+2 n-1}{n^{2}-n+1}-1-\frac{2 n+1}{n^{2}-n+1}-\frac{1}{n^{2}-n+1}-\frac{1}{t\left(n^{2}-n+1\right)} \\
& =\frac{t n-4 t-1}{t\left(n^{2}-n+1\right)} \geq \frac{2 t-1}{t\left(n^{2}-n+1\right)}>0 .
\end{aligned}
$$


It follows from Theorem 2.2 that $F(x)$ has exactly one zero $\rho$ with $|\rho| \geq 1$. Note that $\rho \in \mathbb{R}$. Consequently, $f(x)$ in $(5.3)$ has exactly one zero $\beta$ with $|\beta| \leq 1$, and in fact, $\beta=1 / \rho \in \mathbb{R}$. Hence, if $|\beta|=1$, then $\beta= \pm 1$. However,

$$
f(-1)=(n-2) t+(-1)^{n}>0 \quad \text { and } \quad f(1)=-\left(2 n^{2}+3 n+2\right) t+1<0 .
$$

Thus, $|\beta|<1$. By Descartes' rule of signs, we see from (5.3), that $f(x)$ has exactly one positive real zero $\alpha$. Since $f(1)<0$, we conclude that $\alpha>1$. Therefore, for any prime value $p$ of $t$ with $p>n^{2}-n+1$, we have that $f(x)$ is an anti-Pisot polynomial and $\alpha$ is an anti-Pisot number.

We show next that $f(x)$ is monogenic. Let

$$
g(x)=x^{3}+(2 n+1) x^{2}+\left(n^{2}+2 n-1\right) x+n^{2}-n+1,
$$

and

$$
\begin{aligned}
\widehat{g}(x) & =(n-3) x^{3}+(n-2)(2 n+1) x^{2}+(n-1)\left(n^{2}+2 n-1\right) x+n\left(n^{2}-n+1\right) \\
& =(x+1)(x+n)\left((n-3) x+n^{2}-n+1\right) .
\end{aligned}
$$

Then, assuming that $f(x)$ is irreducible, we apply Theorem 2.12 with $t$ replaced with $-t$ to get

$$
\Delta(f)=(-1)^{\left(n^{2}+7 n\right) / 2} t^{n-1}\left((n-2) t+(-1)^{n}\right)\left(t+(-1)^{n-1} n^{n}\right) \mathcal{Z},
$$

where

$$
\mathcal{Z}=(n-3)^{n-3}\left(4 n^{2}-3 n+22\right) t+(-1)^{n}\left(n^{2}-n+1\right)^{n-1} .
$$

Recall that $n \equiv 0(\bmod 2)$. Then

$$
\begin{aligned}
\delta(t) & :=\frac{\Delta(f)}{(-1)^{\left(n^{2}+7 n\right) / 2} t^{n-1}} \\
& =((n-2) t+1)\left(t-n^{n}\right)\left((n-3)^{n-3}\left(4 n^{2}-3 n+22\right) t+\left(n^{2}-n+1\right)^{n-1}\right) .
\end{aligned}
$$

We desire to apply Corollary 2.8 to $\delta(t)$. Observe that the three linear factors of $\delta(t)$ in (5.6) are distinct. We must also show that $\delta(t)$ has no obstructions. Straightforward ged arguments show that

$$
\operatorname{gcd}\left((n-3)^{n-3}\left(4 n^{2}-3 n+22\right),\left(n^{2}-n+1\right)^{n-1}\right)=1
$$

when $n \equiv 0(\bmod 2)$ and $n \not \equiv 10(\bmod 14)$. Thus, we may proceed as in the proofs of Theorem 1.5 (2) and (3) to conclude that we only have to check for obstructions of $\delta(t)$ at the primes $r \in\{2,3\}$. It is easy to see that $\delta(1) \not \equiv 0(\bmod 4)$. It is also straightforward to show that $\delta(1)+\delta(-1) \not \equiv 0(\bmod 9)$, so that at least one of $\delta(1)$ and $\delta(-1)$ is not 
congruent to $0(\bmod 9)$, for any $n \equiv 0(\bmod 2)$. Thus, $\delta(t)$ has no obstructions, and therefore, by Corollary 2.8, there exist infinitely many primes $p>n^{2}-n+1$ such that $\delta(p)$ is squarefree. For such a prime $p$, it follows that $\Delta(K) \equiv 0(\bmod \delta(p))$. To complete the proof that $f(x)$ is monogenic, we need to show that

$$
\left[\mathbb{Z}_{K}: \mathbb{Z}[\alpha]\right] \not \equiv 0 \quad(\bmod p)
$$

But (5.7) follows immediately from Theorem 2.5 and (5.5), since $f(x)$ is Eisenstein with respect to $p>n^{2}-n+1>n$.

\section{Acknowledgments}

The author thanks the referee for the valuable comments and suggestions.

\section{References}

[1] M.-J. Bertin, A. Decomps-Guilloux, M. Grandet-Hugot, M. Pathiaux-Delefosse and J.-P. Schreiber, Pisot and Salem Numbers, Birkhäuser Verlag, Basel, 1992.

[2] A. Brauer, On algebraic equations with all but one root in the interior of the unit circle, Math. Nachr. 4 (1951), 250-257.

[3] Q. Cheng and J. Zhuang, On certain computations of Pisot numbers, Inform. Process. Lett. 113 (2013), no. 8, 271-275.

[4] H. Cohen, A Course in Computational Algebraic Number Theory, Springer-Verlag, Berlin, 2000.

[5] K. Conrad, Totally ramified primes and Eisenstein polynomials, available at https://kconrad.math.uconn.edu/blurbs/gradnumthy/totram.pdf.

[6] N. J. Fine, Binomial coefficients modulo a prime, Amer. Math. Monthly 54 (1947), $589-592$.

[7] P. W. Haggard and J. O. Kiltinen, Binomial expansions modulo prime powers, Internat. J. Math. Math. Sci. 3 (1980), no. 2, 397-400.

[8] K. G. Hare and N. Sidorov, Conjugates of Pisot numbers, Int. J. Number Theory 17 (2021), no. 6, 1307-1321.

[9] H. A. Helfgott, Square-free values of $f(p), f$ cubic, Acta Math. 213 (2014), no. 1, 107-135. 
[10] C. Hooley, Applications of Sieve Methods to the Theory of Numbers, Cambridge Tracts in Mathematics 70, Cambridge University Press, Cambridge, 1976.

[11] A. Jakhar, S. K. Khanduja and N. Sangwan, Characterization of primes dividing the index of a trinomial, Int. J. Number Theory 13 (2017), no. 10, 2505-2514.

[12] L. Jones, Generating infinite families of monogenic polynomials using a new discriminant formula, Albanian J. Math. 14 (2020), no. 1, 37-45.

[13] Y. Meyer, Algebraic Numbers and Harmonic Analysis, North-Holland Mathematical Library 2, North-Holland Publishing Co., Amsterdam-London; American Elsevier Publishing Co., Inc., New York, 1972.

[14] _ Quasicrystals, Diophantine approximation and algebraic numbers, in: Beyond Quasicrystals (Les Houches, 1994), 3-16, Springer, Berlin, 1995.

[15] R. V. Moody, The Mathematics of Long-range Aperiodic Order, NATO Advanced Science Institutes Series C: Mathematical and Physical Sciences 489, Kluwer Academic Publishers Group, Dordrecht, 1997.

[16] K. Mukunda, Littlewood Pisot numbers, J. Number Theory 117 (2006), no. 1, 106121.

[17] S. Otake and T. Shaska, On the discriminant of certain quadrinomials, in: Algebraic Curves and Their Applications, 55-72, Contemp. Math. 724, Amer. Math. Soc., Providence, RI, 2019.

[18] H. Pasten, The ABC conjecture, arithmetic progressions of primes and squarefree values of polynomials at prime arguments, Int. J. Number Theory 11 (2015), no. 3, $721-737$.

[19] O. Perron, Neue Kriterien für die Irreduzibilität algebraischer Gleichungen, J. Reine Angew. Math. 132 (1907), 288-307.

[20] T. Piezas, F. van Lamoen and E. Weisstein, Plastic constant, available at https://mathworld.wolfram.com/PlasticConstant.html.

[21] C. Pisot, La répartition modulo 1 et les nombres algébriques, Ann. Scuola Norm. Super. Pisa Cl. Sci. (2) 7 (1938), no. 3-4, 205-248.

[22] R. Salem, A remarkable class of algebraic integers: Proof of a conjecture of Vijayaraghavan, Duke Math. J. 11 (1944), 103-108. 
[23] _ Algebraic Numbers and Fourier Analysis, D. C. Heath and Co., Boston, Mass, 1963.

[24] K. Schmidt, On periodic expansions of Pisot numbers and Salem numbers, Bull. London Math. Soc. 12 (1980), no. 4, 269-278.

[25] N. Sidorov and B. Solomyak, On the topology of sums in powers of an algebraic number, Acta Arith. 149 (2011), no. 4, 337-346.

[26] C. L. Siegel, Algebraic integers whose conjugates lie in the unit circle, Duke Math. J. 11 (1944), 597-602.

[27] R. G. Swan, Factorization of polynomials over finite fields, Pacific J. Math. 12 (1962), 1099-1106.

[28] T. Vávra and F. Veneziano, Pisot unit generators in number fields, J. Symbolic Comput. 89 (2018), 94-108.

[29] T. Zaïmi, On small Pisot numbers in a number field, Maghreb Math. Rev. 8 (1999), no. 1-2, 163-167.

Lenny Jones

Professor Emeritus, Department of Mathematics, Shippensburg University,

Shippensburg, Pennsylvania 17257, USA

E-mail address: 1kjone@ship.edu 